\title{
Role of ultrasound in congenital hip dysplasia
}

Ultrasound examination of the hip was first introduced by Graf as a way of assessing surface contour of the pelvis. ${ }^{1}$ It was soon apparent that ultrasound could image the soft tissue components of the infant hip, that is the femoral head and cartilaginous acetabulum, and information could be gained about developmental anatomy and hip location.

Concerns about the specificity and sensitivity of the clinical screening tests for neonatal hip instability prompted an enthusiastic approach by many to the use of ultrasound in the diagnosis and management of congenital dislocation of the hip $(\mathrm{CDH})$. Clarke et al proposed a multiplanar ultrasound examination of the hip with the emphasis on dynamic assessment. ${ }^{2}$ Real time imaging of the hip with 'provocation' of joint instability, effectively a Barlow's test, allows femoral head displacement to be assessed dynamically.

Graf's technique of static measurement relies more on the morphological appearances of the cartilaginous acetabulum. Two angles are calculated. The 'alpha' angle defines the slope of the superior aspect of the osseous acetabulum while the 'beta' angle evaluates the cartilaginous acetabular component. The hip is thus classified into four main types ${ }^{3}$ similar to the classification proposed by Dunn. ${ }^{4}$ The four types effectively define the degree of femoral head displacement and the associated deformation and growth retardation of the acetabular roof. With the static technique the emphasis is placed on an assessment of dysplasia rather than primary hip instability. There have been concerns about interobserver and intraobserver error and the variability in measurement with the morphological technique and consequently the accuracy of hip classification. This is of importance as treatment may or may not be 'prescribed' according to the hip 'type'. However, as the two basic techniques have developed, it has become clear that it is necessary not only to recognise certain essential landmarks but also to make an assessment of hip joint stability.

\section{Ultrasound in the diagnosis and management of CDH}

The clinical tests of Barlow and Ortolani are conventionally used as a screening examination to detect $\mathrm{CDH}$ in the neonate, preferably in the first 24 hours. Despite early optimism about such clinical screening, however, the specificity and sensitivity of the tests have come under scrutiny. ${ }^{5}$ There is a continuing incidence of late established $\mathrm{CDH}$ despite rigorous clinical screening procedures and indeed it is not clear whether clinical screening in some centres does effectively reduce such an incidence. ${ }^{6}$ Additionally there is a high false positive rate in early clinical screening. Barlow originally observed that $60 \%$ of unstable hips will be clinically normal by 1 week of age, ${ }^{7}$ and proponents therefore of immediate abduction splinting for all unstable hips certainly risk over treatment. Splintage may lead to important complications, particularly avascular necrosis, with tragic results if the treated hip instability would have resolved spontaneously without intervention. ${ }^{8}$ Nevertheless the importance of early diagnosis is paramount if conservative splintage treatment is to succeed.
Ultrasound has proved itself as a safe, non-invasive, method of imaging the hip and therefore can be used to resolve the dilemma of whether to splint unstable hips immediately or to delay treatment in the hope that transient instability will resolve. ${ }^{910}$ The policy of a delayed ultrasound examination at 2 weeks in clinically unstable hips will allow treatment to be targeted to those hips that require splintage and thus reduce treatment rates without compromising the results of such treatment. Additionally ultrasound may be used to observe hips on a frequent basis until 6 weeks of age where it is considered that the abnormality is minor or resolving. Delaying treatment after the age of 6 weeks risks a reduction in efficacy and an increased complication rate.

Ultrasound is a very effective aid to the management of $\mathrm{CDH}$ once the diagnosis is confirmed and a decision has been made to proceed to splintage. Indeed the necessity for radiographs and thus exposure to ionising radiation may be eliminated during early management strategies of $\mathrm{CDH}$ involving ultrasound. Hip position may be checked with the child in the splint. It is the author's preference to use the Pavlik harness to treat hip instability. This dynamic splint requires careful management and adjustment and repeated, perhaps weekly, ultrasound studies are employed to confirm hip relocation and treatment progress. Once concentric reduction has been achieved weaning of the harness may proceed, again with ultrasound control. No provocation tests of hip stability are performed in the splint and static views only are obtained. Sonographic evidence of continuing femoral head dislocation despite splintage allows treatment to be abandoned and thus the risk of avascular necrosis is reduced. Ultrasound has also been employed to check the position of the hip in the Von Rosen rigid abduction splint. ${ }^{11}$ After treatment ultrasound is an effective method of reviewing subsequent development of the acetabulum and possible dysplasia and is combined with clinical examination. The appearance of the ossific nucleus in the cartilaginous femoral head (usually delayed in $\mathrm{CDH}$ ) is identified sonographically several weeks before it is visualised radiologically and as it enlarges an acoustic shadow is cast which compromises the ultrasound image. Accordingly conventional radiographic imaging is preferable in the older child with a sizeable proximal femoral ossific nucleus.

The use of ultrasound to define hip position on traction, in the operating theatre, or with the infant in a hip spica cast has not been universally adopted.

\section{Ultrasound in screening for CDH}

The case for incorporating ultrasound into the early management of $\mathrm{CDH}$ is compelling as the subjective tactile interpretations of clinical examination are substituted by static or dynamic sonographic images. The place of ultrasound in the routine screening of all newborns is more controversial. Superficially such an ultrasound based screening programme seems attractive. However, there are substantial and logistical implications which probably preclude such an approach. 
If ultrasound screening is undertaken before the infant leaves hospital there is a captive population but imaging facilities will be required seven days a week. Additionally hip ultrasound at birth will detect a high rate of abnormality. Most sonographers accept that the majority of hips with mild sonographic subluxation or laxity will resolve to normal within four weeks. The high sensitivity of ultrasound in the newborn may therefore lead to correspondingly high treatment rates or follow up attendances or both. Reports have quoted treatment rates of $19.3 \%$ after ultrasound screening. ${ }^{12}$

It is generally accepted that hip ultrasound performed at 2 to 4 weeks provides a more accurate incidence of true hip abnormality as transient or physiological sonographic instability has resolved. However, while universal ultrasound screening at 2 weeks may reduce false positive and treatment rates, this occurs at the expense of a probably higher default rate because of the necessity for recall in the absence of any reduction in the necessary resources.

Consequently strategies have been proposed that embrace the two problems of over diagnosis and logistical difficulty. Delayed selective screening with ultrasound of infants with clinical hip abnormality or risk factors for $\mathrm{CDH}$ (for example, breech delivery, positive family history, foot deformity) reduces the screened population and allows treatment options to be delayed and targeted correctly. Such an approach has, however, been reported not to reduce the prevalence of late cases of $\mathrm{CDH},{ }^{13}$ and it is apparent, therefore, that a protocol of selective ultrasound screening is dependent upon vigorous clinical screening and careful selection of risk factors. The experience in Southampton suggests that the two can be combined with ultrasound to reduce the incidence of late dislocation $(0 \cdot 211 / 1000)$, treatment rate $(4 \cdot 4 / 1000)$, and screened population $(7 \%)$. The problem remains, however, that not all abnormal hips are detected in such a scheme.

\section{Conclusion}

There is now no doubt that ultrasound examination of the infant hip is reliable and accurate. It is a relatively new technique and as such many new issues have been raised. As it becomes more universally accepted it is important that prospective trials provide data to ensure that errors in diagnosis and subsequent treatment of $\mathrm{CDH}$ are not compounded. It is to be hoped that a consensus may be achieved so that a standard examination can be performed incorporating static and dynamic views but the technique remains operator dependent. Experience is necessary and close cooperation between clinicians and imagers is mandatory whether they be paediatricians, orthopaedic surgeons, or radiologists.

Ultrasound is the imaging method of choice in the diagnosis and management of early neonatal hip instability. Its role in screening is yet to be defined but the use of ultrasound to augment primary clinical screening seems to confer benefit and as such should be increasingly adopted. Hopefully with increasing knowledge of the natural history of $\mathrm{CDH}$ the tragedy of late presenting cases will be addressed.

Department of Orthopaedic Surgery,

N M P CLARKE

Southampton General Hospital,

Tremona Road,

Southampton SO9 $4 X Y$

1 Graf $R$. The diagnosis of congenital hip joint dislocation by the ultrasound combound treatment. Arch Orthop Trauma Surg 1980; 97: 117-33.

Clarke NMP, Harcke HT, McHugh P, Lee MS, Borns PF, MacEwen GD

Real-time ultrasound in the diagnosis of congenital dislocation and dysplasia of the hip. $\mathcal{F}$ Bone foint Surg Br 1985; 67: 406-12.

3 Graf R. Classification of hip joint dysplasia by means of sonography. Arch Orthop Trauma Surg 1984; 102: 248-55.

4 Dunn PM. The anatomy and pathology of congenital dislocation of the hip. Clin Orthop 1976; 119: 23-7.

5 Leck I. An epidemiological assessment of neonatal screening for dislocation of the hip. $\mathcal{F} R$ Coll Physicians Lond 1986; 20: 56-61.

6 Clarke NMP. Diagnosing congenital dislocation of the hip. BMf 1992; 305:

7 Barlow TG. Early diagnosis and treatment of congenital dislocation of the hip. F Bone foint Surg Br 1962; 44: 292-301.

8 Langkamer VG, Clarke NMP, Witherow P. Complications of splintage in congenital dislocation of the hip. Arch Dis Child 1991; 66: 1322-5.

9 Gardiner HM, Dunn PM. Controlled trial of immediate splinting versus ultrasonographic surveillance in congenitally dislocated hips. Lancet 1990 336: 1553-6.

10 Clarke NMP. Sonographic clarification of the problems of neonatal hip instability. $\mathcal{f}$ Pediat Orthop 1986; 6: 527-32.

11 Dahlstrom H, Friberg S, Oberg L. Stabilisation and development of the hip after closed reduction of late CDH. $\mathcal{f}$ Bone foint Surg Br 1990; 72: 186-9.

12 Tonnis D, Storch K, Ulbrich H. Results of newborn screening for $\mathrm{CDH}$ with and without sonography and correlation of risk factors. $\mathcal{F}$ Pediatr Orthop 1990; 10: 145-52.

13 Clarke NMP, Clegg J, Al-Chalabi AN. Ultrasound screening of hips at risk for $\mathrm{CDH}$. Failure to reduce the incidence of late cases. $\mathcal{f}$ Bone foint Surg Br 1989; 71: 9-12. 